\title{
Investigation of the Effect of an Increased Supervisory Signal Power in a High-Loss Loopback Monitoring System
}

\author{
Mousaab M. Nahas \\ Electrical and Computer Engineering, Faculty of Engineering - North Jeddah, King Abdulaziz \\ University, KSA \\ E-mail: mnahas1@kau.edu.sa \\ Keith J. Blow \\ School of Engineering \& Applied Science, Aston University, Birmingham, UK \\ E-mail: k.j.blow@aston.ac.uk
}

\begin{abstract}
We present experimental results for the effect of an increased supervisory signal power in a high-loss loopback supervisory system in an optically amplified WDM transmission line. The paper focuses on the investigation of increasing the input power for the supervisory signal and the effect on the copropagating WDM data signals using different channel spacing. This investigation is useful for determining the power limitation of the supervisory signal if extra power is needed to improve the monitoring. The paper also shows the effect of spacing on the quality of the supervisory signal itself due to interaction with adjacent data signals.
\end{abstract}

\section{Keywords}

Long-haul undersea systems, WDM communication systems, optical line monitoring, optical supervisory systems.

\section{Introduction}

Submarine fiber links require remote monitoring in real time to instantaneously locate faults in the transmission line. Such instantaneous determination of the fault allows instant repair activity to be performed on the system. In the absence of electrical regeneration, which can provide distributed 
feedback on the BER, optical techniques are used. The most famous optical line monitoring technique, which was first developed in the late eighties, uses coherent optical time domain reflectometry (COTDR) to monitor optically amplified submarine cable systems [1]-[4]. The main disadvantage of this method is the cost, largely related to the use of optical coherent detection. The other disadvantage is that it does not work effectively for a long-haul fiber span as the backscattered signal needs to bypass the optical amplifiers through couplers because of the optical isolators used to prevent lasing in the amplifiers [2].

To overcome the above drawbacks, a high-loss loopback supervisory system was first proposed in 1996 to provide low cost, in-service and out-of-service monitoring of long-haul optically amplified fiber links [5]. This method is based on setting up a simple, passive high-loss optical loopback circuit at each repeater to connect between the existing two anti-directional fibers as shown in Fig. 1. The high-loss loopback circuits can tap off a portion of any propagating signal, attenuate it and return it back to the transmit terminal. Bi-directional line monitoring of a supervisory signal is thus achieved. The fault location is deduced from the level and delay of the supervisory signal, as no digital information is returned. Typically the fault is anticipated to be due to low gain in an Er amplifier.

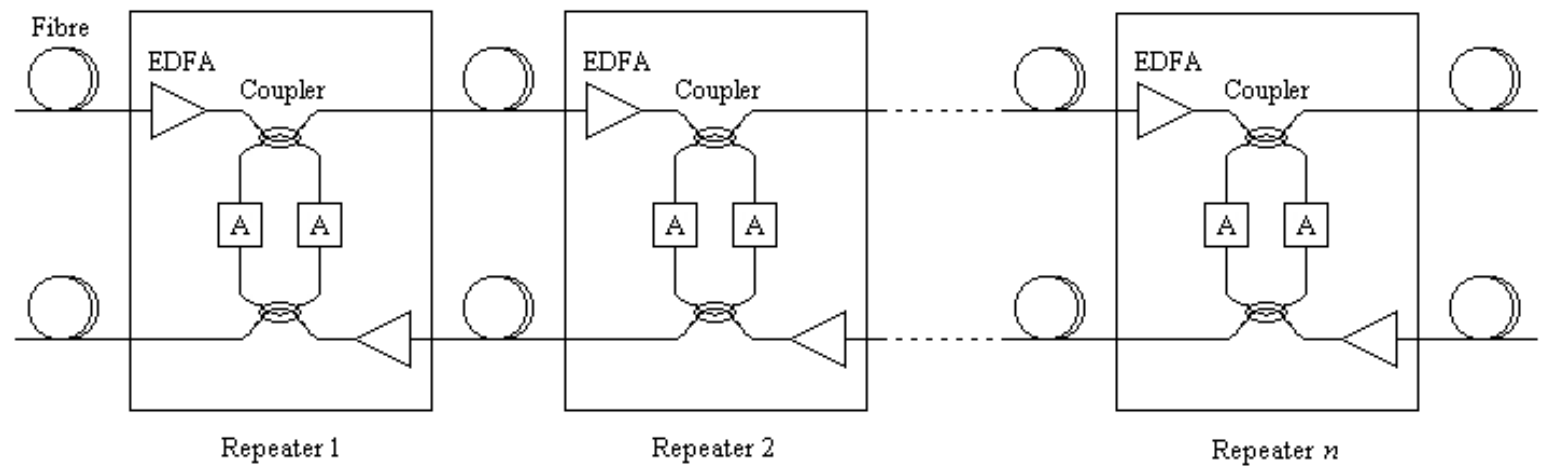

Fig. 1. Schematic diagram of the high-loss loopback circuits.

Experimental results of the above approach have been presented [6] using line monitoring equipment (referred to as LME) to monitor one propagation path. Successful monitoring was accomplished 
through producing an appropriate low-power LME signal and recovering this signal in a satisfactory measurement time after being transmitted over $4600 \mathrm{~km}$ and attenuated by $45 \mathrm{~dB}$ loss in a loopback circuit. To achieve this, the paper used the so called out-of-band LME system [7] in which the LME wavelength is different from the traffic wavelengths rather than the in-band scheme proposed in [8]. Such an out-of-band approach allowed us to use [6] a simplified LME receiver apparatus to extract the LME signal, resulting in an in-service measurement time to be in the order of 1 minute rather than $\sim 3$ hours as in [8]. Moreover, our previous work [6] proved that an increment in the LME launch power results in a linear improvement in the loopback attenuation with a slope $\sim 1$. This allowed us to make the LME receiver work with $45 \mathrm{~dB}$ of loopback attenuation which is a typical value according to [7] and $[8]$.

In practice, the LME receiver always works better if the launch power is increased although the original approach aimed to keep the supervisory signal power as low as possible. However, in case of the dramatic drop of one or more than one EDFA gain, it might be necessary to make use of higher power levels to improve the detection and to ensure recovery of the returned LME signals. Nevertheless, this can be performed only if an increment on the supervisory signal power would not impair the accompanying data traffic signals. Therefore, this paper is devoted to presenting an experimental investigation of increasing the input power for the LME signal and its effect on the copropagating WDM data traffic signals for different channel spacing using the out-of-band LME approach as demonstrated in [6]. This investigation enables identifying the power limitation of the LME signal in WDM transmission systems. (Note that the counter-propagating data are not affected by the looped back signals and noise since they are attenuated by $45 \mathrm{~dB}$ in the loopback circuit). We also use a different dispersion map in the optical system being monitored.

\section{Experimental setup}




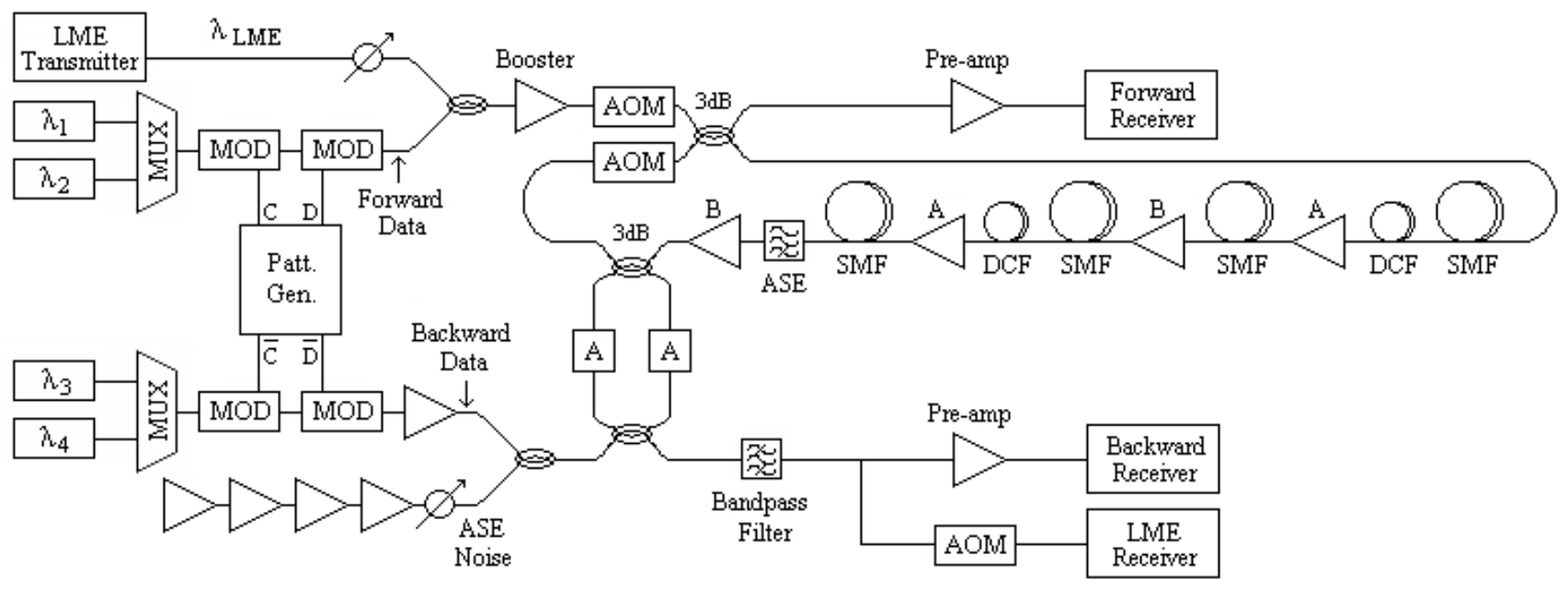

Fig. 2. Experimental setup.

The experimental setup of this work is shown in Fig. 2. It uses a single out-of-band LME to monitor one traffic direction using a high-loss loopback circuit in a recirculating loop. Full details of the system can be found in [6].

The recirculating loop consists of two fiber sections using a symmetric dispersion map in each section rather than a periodic map as used in [6]. This configuration reduces the accumulated dispersion over the fiber spans and thus improves the loop behavior so that the $Q$-value for the forward data signals is now measurable and can be used instead of the OSNR [6]. The first section of the loop has $40.7 \mathrm{~km}$ SMF followed by $16.5 \mathrm{~km}$ DCF with $-1383 \mathrm{ps} / \mathrm{nm}$ dispersion, which is then followed by another SMF of $41.8 \mathrm{~km}$. In the second section, $42.9 \mathrm{~km}$ SMF is followed by $15.2 \mathrm{~km}$ DCF with $-1387 \mathrm{ps} / \mathrm{nm}$ dispersion, and then $38 \mathrm{~km}$ of SMF. The losses of the fiber spans are compensated by using two Cband EDFAs in each section as described in [6]. The loopback circuit is set up at the end of the recirculating loop so that it monitors the performance of the last EDFA [6]. Monitoring different repeaters is emulated by increasing the number of recirculations. Although the loopback is implemented every four amplifiers compared to the real system implementation shown in Fig.1, the setup is still simulating the real effect over those repeaters seen every $195 \mathrm{~km}$, where other repeaters are not necessarily examined. 
The backward path carries two WDM channels modulated with inverted clock and data as compared to the forward path. The backward channels pass through an Er amplifier chain to set the backward propagating noise level [6] to match the transmission distance used in the forward direction. Although the backward data are injected at constant OSNR, which in real life will not be the case, the OSNR values used here are nevertheless realistic as long as the backward noise is chosen to match the noise of the forward direction. A tunable band-pass filter with $0.24 \mathrm{~nm}$ bandwidth and $7 \mathrm{~dB}$ insertion loss extracts the supervisory wavelength for detection and analysis. The filter is also used to demultiplex the backward data signals for measurement. The LME signal is extracted by an AOM as before [6].

\section{Results and analysis}

\subsection{Forward data traffic performance}

To investigate the effect of increasing the LME signal power on the co-propagating WDM data, the performance of the forward channels must be examined first via the measurement of either $Q$-value or BER. Fig. 3 shows the $Q$-value measurements as a function of transmission distance for the channel at $\lambda_{2}=1557.4 \mathrm{~nm}$ that is adjacent to the LME signal using $0 \mathrm{dBm}$ launch power in both data signals. Note that the $Q$-value measurements were based on the means and standard deviations of the marks and spaces taken from the sampling oscilloscope. The data signals propagate over $\sim 3300 \mathrm{~km}$ before Q drops below 6, and this transmission distance is therefore the upper limit of this system. Although it was possible to obtain acceptable OSNR measurements at longer distances $(>4000 \mathrm{~km})$, considerable distortion was noticed on the signal due to PMD and nonlinearities beyond $3300 \mathrm{~km}$. 


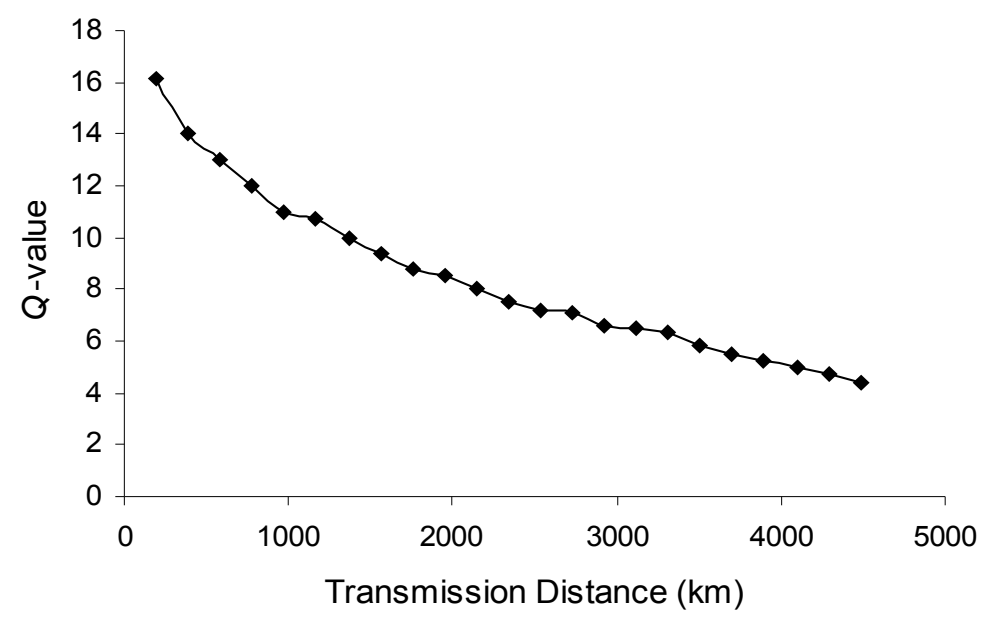

Fig. 3. $Q$-value versus transmission distance for data signal $\lambda_{2}=1557.4 \mathrm{~nm}$ using $0 \mathrm{dBm}$ launch power.

3.2 Line monitoring resultsHaving established acceptable performance of the transmission system we now evaluate the supervisory system as in [6]. The LME system transmits a supervisory pulse and then detects the returned portion of this pulse. The receiver has to recover the LME signal from the high noise levels generated by the backward traffic. The LME pulse is transmitted as a $2 \mathrm{MHz}$ subcarrier and the detection of its carrier envelope is accomplished through an IQ demodulator used at the LME receiver[6]. The amplitude of the received carrier is then proportional to $\left(\mathrm{I}^{2}+\mathrm{Q}^{2}\right)^{1 / 2}[7]$. The LME pulse of different repeaters is displayed at different times according to the repeater distance that corresponds to the number of recirculations in the loop.

The line monitoring results are shown in Fig. 4, which presents successful LME pulse recovery up to the maximum transmission length of $3300 \mathrm{~km}$ using the typical $45 \mathrm{~dB}$ loopback attenuation. More specifically, the figure shows the eSNR of the LME signal which had a lauch power of -8 $\mathrm{dBm}$ (corresponding to $-2 \mathrm{dBm}$ into the loop $\mathrm{AOM}$ ). These eSNR measurements are obtained using the method described in [6]: 


$$
\mathrm{eSNR}=\frac{\operatorname{Ave}\left(\mathrm{I}^{2}+\mathrm{Q}^{2}\right)}{\operatorname{Var}(\mathrm{I}, \mathrm{Q})}
$$

where the $\mathrm{I}^{2}+\mathrm{Q}^{2}$ peak is averaged over a small region around the maximum value, and the $\mathrm{I}$ and $\mathrm{Q}$ variance is measured over a wider region in the zero level as shown in Fig. 5.

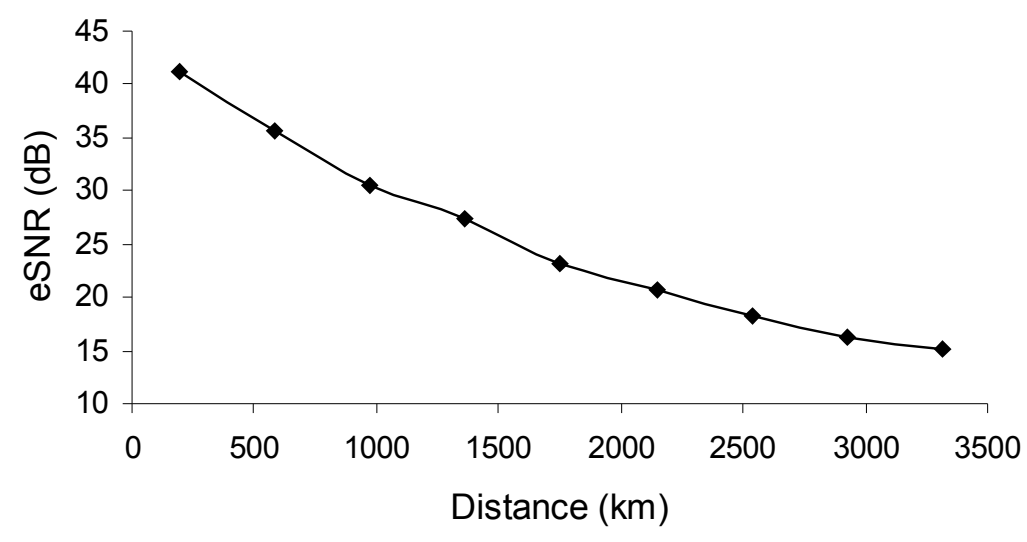

Fig. 4. eSNR measurements versus propagation distance for the LME signal using $-8 \mathrm{dBm}$ launch power and $45 \mathrm{~dB}$ loopback attenuation.

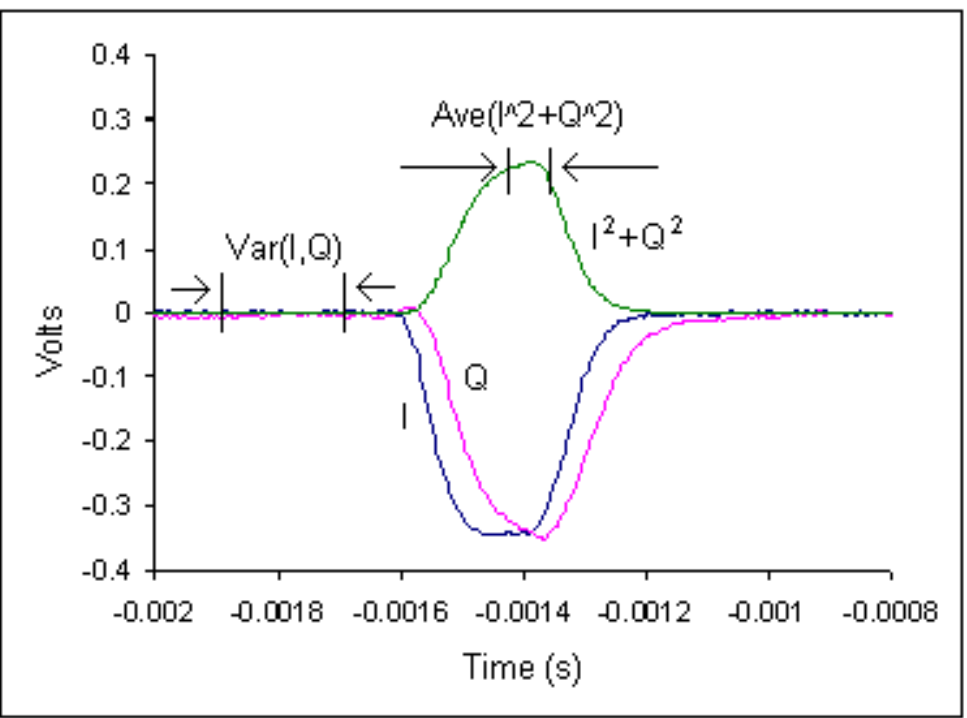

Fig. 5. eSNR measurement method. 
In practice, the eSNR values shown in Fig. 4 can be improved if the LME signal launch power increases above $-8 \mathrm{dBm}$. This is absolutely required in case that, for some reason (e.g. longer distance), the returned noise has degraded the LME signal such that it is undetectable by the supervisory receiver. The next section discusses the effect of increasing the supervisory signal power and the limitations in WDM systems.

\subsection{An increased LME signal power effect}

The effect of the LME signal is tested by simply increasing the power of the LME signal and observing the effect on the $Q$-value of the adjacent data signal that is in practice most affected. To do this, the data signal power is fixed at $0 \mathrm{dBm}$ and the test is run over $3300 \mathrm{~km}$ (the maximum propagating distance for our system). Note that increasing the LME power would increase the total average power launched into the fiber, thus the gains of the loop EDFAs are re-adjusted for each measurement.

Fig. 6 presents the observed $Q$-value as a function of the LME signal power measured at the input to the first loop fiber, using a transmission distance of $3300 \mathrm{~km}$. It can be seen that the performance of the forward data signal (adjacent to the LME signal) starts to be affected if the LME signal power exceeds the data signal power by $4 \mathrm{~dB}$ (obtained from the turning point of the curve in Fig. 6). Since the OSNR is maintained for the data signals and the system works in the zero average dispersion region, the only cause for degradation would be the inter-channel crosstalk caused by nonlinear interaction. Although data signals at $10 \mathrm{Gbit} / \mathrm{s}$ and $125 \mathrm{GHz}$ spacing need higher power for noticeable cross-phase modulation (XPM) and four-wave mixing (FWM) over such a distance, the LME signal with $4 \mathrm{dBm}$ power has considerable nonlinear interaction with its adjacent data signal because it has a $500 \mu \mathrm{s}$ envelope that fills most of the recirculating loop round-trip time. This makes the LME signal interact with the data signal continuously over a large fraction of the recirculating loop thus significant power transfer between the signals is detected causing a degradation in the return to zero (RZ) signal quality. This situation should never occur in reality as the LME signal is intended to be much lower than the data signals with at least $6 \mathrm{~dB}$ difference. However, this is useful from a system viewpoint as the data 
traffic will not be affected by an increase in the LME power even when it becomes comparable to the data signal power. On the other hand, the operator can use an increased supervisory signal power comfortably in case of bad LME pulse recovery as in [6], in which the LME launch power increased from $-16 \mathrm{dBm}$ up to $-8 \mathrm{dBm}$, keeping $8 \mathrm{~dB}$ difference from the data signals.

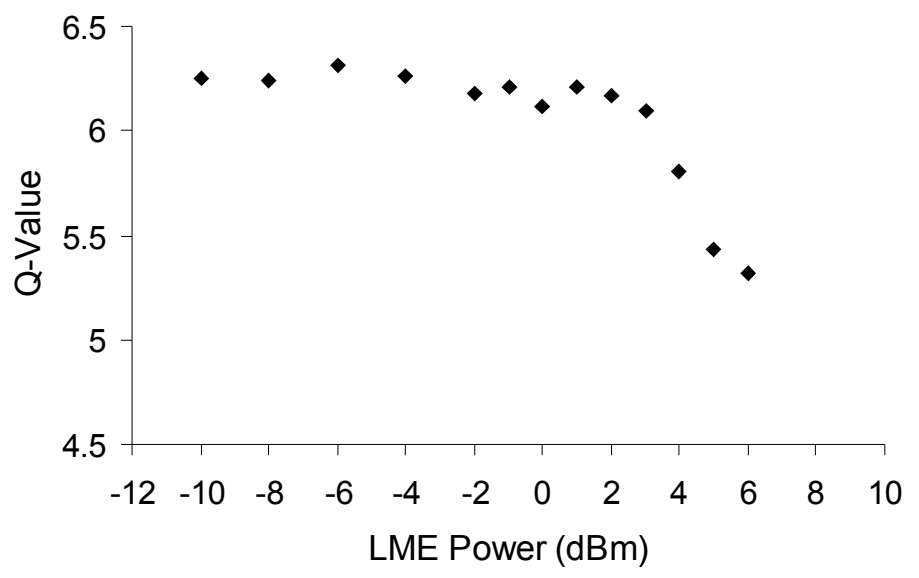

Fig. 6. $Q$-value of the forward data signal $\lambda_{2}=1557.4 \mathrm{~nm}$ versus LME launch power, measured over $3300 \mathrm{~km}$ propagation distance.

For generalization, the same test was repeated for a different spectral position of the LME channel through swapping the LME wavelength with the adjacent signal wavelength thus the LME signal is placed in the middle of the two data signals, keeping $1 \mathrm{~nm}$ spacing. As a result, a similar curve has been obtained and it was found that both signals would be affected if the LME signal power reaches $\sim 4$ $\mathrm{dBm}$.

\subsection{Spacing effect on data signals}

The above results were all obtained for $1 \mathrm{~nm}(125 \mathrm{GHz})$ spacing between the LME and data signals. In practice, if the spectral band is fully loaded with WDM signals being separated by $1 \mathrm{~nm}$ for example, it is then required to have the LME channel spaced from the adjacent data signals by less than $1 \mathrm{~nm}$ (ideally by $0.5 \mathrm{~nm}$ ). Therefore, it is necessary to examine the performance of data signals with reduced spacings. The results of this test are shown in Fig. 7 using $3300 \mathrm{~km}$ transmission distance again. For 
comparison, we plotted the LME power at which the data signals start to be affected (i.e. the turning point in Fig. 5) versus spacing.

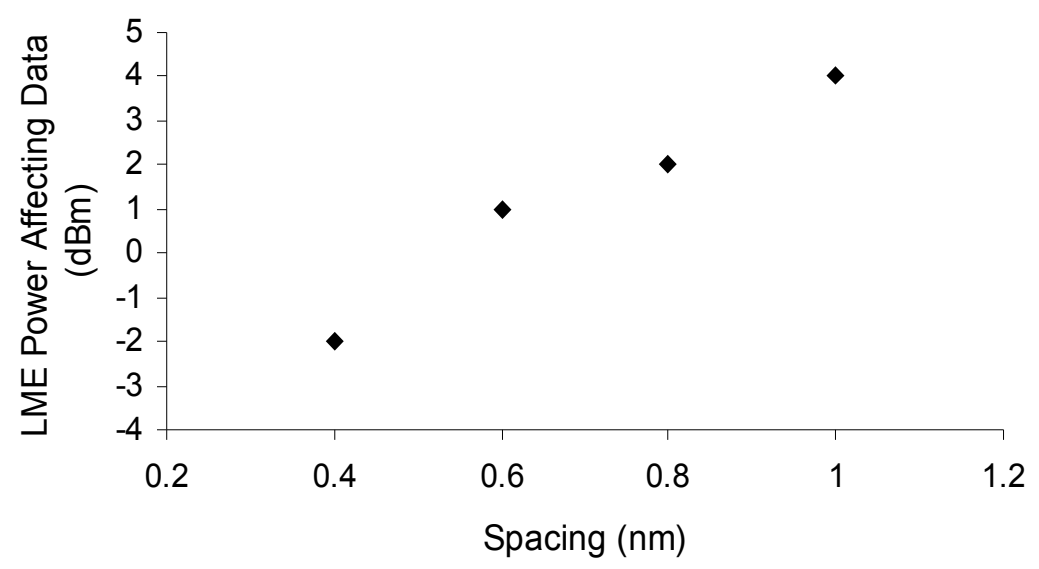

\section{Fig. 7. LME power turning point versus spacing between the LME signal and adjacent data signals.}

It is noticed that the turning point degrades as the LME signal gets closer to the data signals due to more inter-channel crosstalk caused by XPM and FWM. However, the LME signal still requires high power to affect the data traffic performance. For instance, if the spacing is $0.4 \mathrm{~nm}$, the LME signal needs at least $-2 \mathrm{dBm}$ to affect the adjacent data signals whose launch power is $0 \mathrm{dBm}$. In other words, the LME signal will have no effect on the data signals provided its power is lower by $2 \mathrm{~dB}$, which should easily be achieved. However, this result still allows the operator to use an increased LME power if necessary to achieve higher loopback attenuation even with a small spacing such as $0.4 \mathrm{~nm}$. In reality, the results of $0.4 \mathrm{~nm}$ spacing are interesting as WDM signals are typically spaced apart by 0.8 $\mathrm{nm}$, and the LME signal is supposed to be placed halfway between any two WDM data wavelengths.

In fact, the results obtained so far are true for wavelengths operating in the zero average dispersion region where nonlinear effects are high. This means that if the wavelengths are chosen to be sufficiently far away from this region, the data signals can be tolerant to higher LME power. However, such an option is not considered here as we are studying the power limitation of the LME signal based on the worst case conditions. 


\subsection{Spacing effect on LME signal}

The previous results assumed that there is no effect from the data signals on the LME signal. To fully determine the limitations of the power and spacing for our monitoring system, we now examine the effect of on the separation on the LME signal itself over the same propagation distance $(3300 \mathrm{~km})$. The results of this test are presented in Fig. 8 using $-8 \mathrm{dBm}$ launch power for the LME signal and $0 \mathrm{dBm}$ for the data signals as originally proposed. Since the quality of the LME signal can only be assessed through eSNR (as no data are carried), Fig. 8 shows the eSNR measurement of the returned LME signal versus spacing to the adjacent data signals when the LME is on the side, and when it is in the middle. The penalty on the LME signal performance is largest at $0.4 \mathrm{~nm}$ in both cases due to considerable nonlinear interaction with the data wavelengths, but the LME signal is still being recovered. To compensate for this penalty, it is possible to take advantage of the results shown in the previous section through increasing the LME signal power entering the fiber. This would ensure good detection at the LME receiver without harming the data traffic signals.

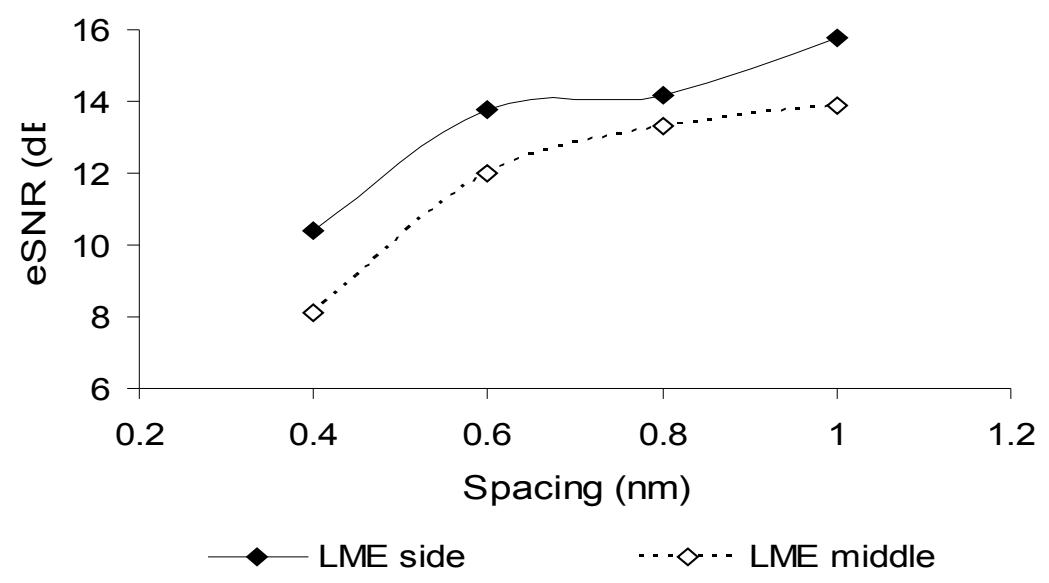

Fig. 8. eSNR of the LME signal versus spacing between the LME signal and adjacent data

\section{Conclusions} signals.

This paper studies the impact of an optical LME system on the performance of a long haul, optically amplified WDM communication system. We demonstrated experimentally the effect of increasing the input power of the supervisory signal and the effect on the co-propagating WDM data traffic using 
different channel spacings. It was found that data traffic is not affected by the increase in the supervisory signal power even when its power becomes comparable to the data signal power. This in fact allows using an increased supervisory signal power to improve detection if required. The opposite effect has also been studied i.e. the effect of the data signals on the co-propagating LME signal for a reduced spacing. It was found that a considerable degradation in the LME eSNR would appear if the spacing between the LME signal and its adjacent signals is less than $0.6 \mathrm{~nm}$, although the LME signal can still be detected down to $0.4 \mathrm{~nm}$ spacing. This degradation can be overcome by increasing the supervisory signal power especially in case that the supervisory pulse is lost due to the small channel spacing.

\section{Acknowledgments}

The authors thank Azea Networks for financial support and Stuart Barnes, Steve Webb, Stephen Desbrulais, John Ellison, Joerg Schwartz and Robin Ibbotson for helpful discussions and suggestions during the course of this work.

\section{References}

1. J. P. King, D. F. Smith, K. Richards, P. Timson, R. E. Epworth, and S. Wright, "Development of a coherent OTDR instrument". Journal of Lightwave Technology, vol. 5, pp. 616-624, 1987.

2. Y. Horiuchi, S. Ryu, K. Mochizuki, and H. Wakabayashi, "Novel coherent heterodyne optical time domain reflectometry for fault localization of optical amplifier submarine cable systems". IEEE Photonics Technology Letters, vol. 2, pp. 291-293, 1990.

3. Y. Sato, and K. Aoyama, "Optical time domain reflectometry in optical transmission lines containing in-line Er-doped fiber amplifiers”. Journal of Lightwave Technology, vol. 10, pp. 78-83, 1992.

4. P. Kim, H. Yoon, N. Park,a, J. Seo, K. Jeong, K. Ryoo, K. Lee, J. Blow, and S. Fleming, "Novel in-service supervisory scheme for the amplified WDM link with modified optical time domain reflectometry". Optical Fiber Technology, vol. 8, pp. 139-145, 2002.

5. C. D. Anderson, "Supervisory apparatus for optical transmission system", U.S. Patent 5,825,515, (filed May 1996).

6. M. M. Nahas, and K. J. Blow, "Monitoring long distance WDM communication lines using a highloss loopback supervisory system", Optics Communications, vol. 285, pp. 2620-2626, 2012.

7. S. Desbruslais, J. Ellison, and D. Powell, "Pulsed high loss loop back signaling scheme", UK Patent GB 2413447 B, (filed April 2005).

8. S. Desbruslais, J. Ellison, and D. Powell, "Short pulse loop back supervisory system", UK Patent GB 2413447 A, (filed April 2005). 